\title{
Digital microfluidics: Magnetic transportation and coalescence of sessile droplets on hydrophobic surfaces
}

\author{
Md Rifat Hassan, Jie Zhang, and Cheng Wang* \\ Department of Mechanical and Aerospace Engineering, Missouri University of Science and \\ Technology, 400 W. 13th St., Rolla, Missouri, 65409, USA \\ E-mail: wancheng@mst.edu
}

\section{Supporting Information Available}

\section{Mass conservation of droplet}

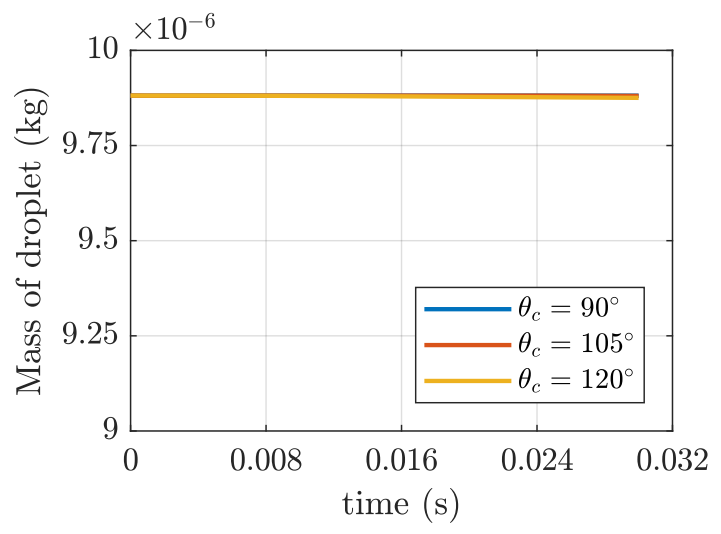

Figure 1: Mass of a sessile droplet under various wetting conditions.

In order to demonstrate the clear advantage of phase field (PF) method over other numerical methods i.e., level set (LS) method, volume of fluid (VOF) method in terms of conserving 
the mass of a droplet in fluid domain, an investigation on the mass of a droplet is carried out under various wetted wall conditions. Figure 1 illustrates the time evolution of the mass of a sessile droplet at different contact angles, which clearly indicates that irrespective of different boundary conditions, the droplet mass is conserved throughout the whole process in numerical simulations.

\section{Grid independence check}

Table 1: Maximum mesh element size in the computational domain

\begin{tabular}{|c|c|c|c|c|}
\hline Mesh 1 & Mesh 2 & Mesh 3 & Mesh 4 & Mesh 5 \\
\hline $0.2 R_{0}$ & $0.1 R_{0}$ & $0.075 R_{0}$ & $0.06 R_{0}$ & $0.05 R_{0}$ \\
\hline
\end{tabular}

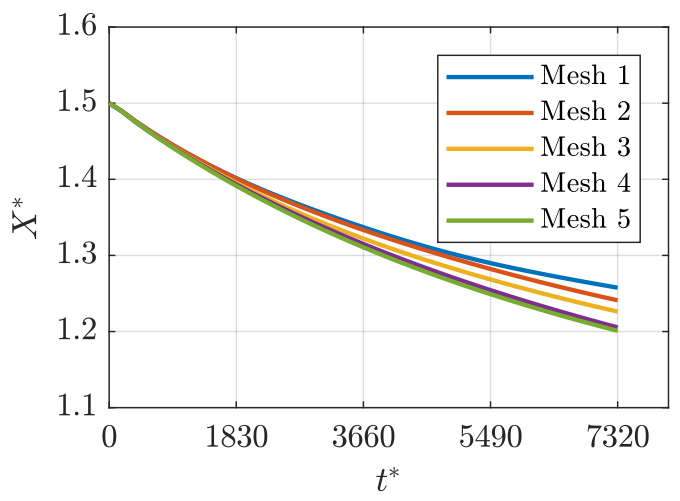

Figure 2: Grid independence test: time evolution of the magnetic field induced migration of a sessile droplet along the horizontal direction $X^{*}$ for different mesh configurations at $\mathrm{Bo}_{m}=0.145$ and $\theta_{c}=150^{\circ}$.

Figure 2 represents the time evolution of the magnetic field induced migration of a sessile droplet along the horizontal direction $X^{*}$ for several mesh configurations at $\mathrm{Bo}_{m}=0.145$ and $\theta_{c}=150^{\circ}$, which are tabulated in Table 1 , and it can be seen that when the maximum mesh size in the computational domain is less than $0.06 R_{0}$, the migration results tend to overlap with each other, while traversing the same amount of distance along the horizontal direction from initial position in the time under consideration. As a result, using more refined meshes in the computational domain would only lead to minimal changes in the coalescence time, 
while demonstrating similar coalescence behavior under the same conditions at the expense of high computational costs. Considering the computational cost, we have decided to use $0.05 R_{0}$ as the magnitude of the maximum mesh element size in the domain under consideration, which eventually saves a reasonable amount of computational time, while providing accurate estimation of dynamic droplet interface during the simulations throughout the paper. 